\title{
Metal nanofibrils embedded in long free- standing carbon nanotube fibers with a high critical current density
}

Hokyun Rho ${ }^{1,2}$, Min Park', Mina Park', Junbeom Park', Jiyoon Han', Aram Lee', Sukang Bae', Tae-Wook Kim', Jun-Seok Ha², Seung Min Kim (1)', Dong Su Lee ${ }^{1}$ and Sang Hyun Lee (1)

\begin{abstract}
With the growth of nanoelectronics, the importance of thermal management in device packaging and the improvement of high-current-carrying interconnects/wires for avoiding the premature failure of devices have been emphasized. The heat and electrical transport properties of the bulk may not be valid in the characterization of a material at the nanometer level, because the phenomena that occur at the interfaces and grain boundaries become dominant. The failure mechanism of bulk metal interconnects has been understood in the context of electromigration; however, in nanoscale materials, the effect of the heat dissipation that occurs at the nanointerfaces may play an important role. Here, we report the preparation of continuous carbon nanotube (CNT)-Cu composite fibers that possess Cu nanofibrillar structures with a high current-carrying capacity. Various-shaped CNT-Cu microfibers with different Cu grain morphologies were produced via Cu electroplating on continuous CNT fibers. Cu fibril structures were embedded in the voids inside the CNT fiber during the early stage of electrodeposition. The temperaturedependent and magnetic field-dependent electrical properties and the ampacity of the produced CNT-Cu microfibers were measured, and the failure mechanism of the fiber was discussed. The interconnection of Cu nanograins on the surface of the individual CNTs contributed to the enhancement in the charge-carrying ability of the fiber. The effective ampacity of the Cu nanofibrils was estimated to be $\sim 1 \times 10^{7} \mathrm{~A} / \mathrm{cm}^{2}$, which is approximately 50 times larger than the ampacity measured for a bulk Cu microwire.
\end{abstract}

\section{Introduction}

The enhancement of the current-carrying capacity is a crucial issue for many applications, such as integrated microelectronic/optoelectronic devices ${ }^{1}$, high-power devices $^{2,3}$ and power transmission cables. Hot carriers that are generated under a high current bias significantly degrade the performance of the devices and/or reduce the lifetimes of the cables and circuits ${ }^{4}$. As devices shrink to smaller dimensions, selecting materials for electrical

\footnotetext{
Correspondence: Dong Su Lee (d.s.lee@kist.re.kr) or Sang Hyun Lee (sanghyun. lee@kist.re.kr)

${ }^{1}$ Institute of Advanced Composite Materials, Korea Institute of Science and Technology, Wanju-gun, Jeonbuk 55324, Republic of Korea

${ }^{2}$ Optoelectronics Convergence Research Center, Chonnam National University, Gwangju 61186, Republic of Korea

These authors contributed equally: Hokyun Rho, Min Park.
}

components and arranging them into integrated circuitry become important challenges. In microscopic interconnect components, electron scattering at the interfaces and grain boundaries play a dominant role in charge carrier transport ${ }^{5}$ and heat generation ${ }^{6}$. However, the heat dissipation, cross-effects of Joule heating and electromigration that occur on the nanoscale have not been actively elucidated ${ }^{7}$. Enhancing the current-carrying capacity of interconnect materials composed of metal nanostructures and nanocarbon materials, such as carbon nanotubes (CNTs) and graphene, may be a solution ${ }^{8-12}$. In these approaches of utilizing metal-carbon compositions, systematic studies that provide a clear understanding of the synergetic effects are still lacking.

\section{(c) The Author(s) 2018}

(c) (i) Open Access This article is licensed under a Creative Commons Attribution 4.0 International License, which permits use, sharing, adaptation, distribution and reproduction BY in any medium or format, as long as you give appropriate credit to the original author(s) and the source, provide a link to the Creative Commons license, and indicate if changes were made. The images or other third party material in this article are included in the article's Creative Commons license, unless indicated otherwise in a credit line to the material. If material is not included in the article's Creative Commons license and your intended use is not permitted by statutory regulation or exceeds the permitted use, you will need to obtain permission directly from the copyright holder. To view a copy of this license, visit http://creativecommons.org/licenses/by/4.0/. 
Herein, we present a systematic study of the electrical and thermal interplay at the $\mathrm{CNT}-\mathrm{Cu}$ interface. We employed a practical approach to obtain continuous $\mathrm{CNT}-\mathrm{Cu}$ composite fibers. These continuous CNT fibers were used as cathodes during $\mathrm{Cu}$ electroplating processes. The amount of deposited $\mathrm{Cu}$ in the resulting structures varied. The $\mathrm{CNT}-\mathrm{Cu}$ fibers were categorized into five groups. The fibers that contain a small amount of $\mathrm{Cu}$ for nucleation are in the first group. As the amount of $\mathrm{Cu}$ deposited increases, the fibers change to core-shell-type cylinders with a thick outer $\mathrm{Cu}$ layer. The measured ampacity of the $\mathrm{CNT}-\mathrm{Cu}$ fibers increases with increasing amount of deposited $\mathrm{Cu}$ in the early stage. However, afterwards, the ampacity begins to saturate and slightly decreases in the thick core-shell type samples. The highest ampacity of the CNT fibers reaches $\sim 4.89 \times 10^{5} \mathrm{~A} / \mathrm{cm}^{2}$, in which $\mathrm{Cu}$ conduction paths of connected nanograins are formed. This ampacity is already 30 times higher than the measured value of pristine CNT fibers. The temperaturedependent resistivity and magnetoresistance (MR) results indicate that the electroplated $\mathrm{Cu}$ has different properties than bulk $\mathrm{Cu}$, such as a lower residual resistivity ratio (RRR) and MR. Additionally, we found that the embedded $\mathrm{Cu}$ forms continuous nanofibrillar structures that dominate the electrical conduction at high temperatures. We extracted the effective area of the $\mathrm{Cu}$ fibrils. Using this value and the current-voltage characteristics, we obtained the effective ampacity of the $\mathrm{Cu}$ fibrils $\left(\sim 1 \times 10^{7} \mathrm{~A} / \mathrm{cm}^{2}\right)$, which is approximately 50 times higher than the measured value of a bulk $\mathrm{Cu}$ wire.

\section{Materials and methods}

\section{Fabrication of the CNT fibers}

In this work, continuous CNT fibers were spun from CNT aerogels that were formed in a chemical vapor deposition (CVD) reactor $^{13}$. Methane (carbon source, $2.045 \times 10^{-3} \mathrm{~mol} / \mathrm{min}$ ), ferrocene (catalyst, $1.668 \times 10^{-6}$ $\mathrm{mol} / \mathrm{min}$ ) and thiophene (promoter, $3.962 \times 10^{-6} \mathrm{~mol} /$ min) were injected into the gas phase with hydrogen as the carrier gas $\left(3.931 \times 10^{-2} \mathrm{~mol} / \mathrm{min}\right)$. The injection lines were also heated to approximately $373 \mathrm{~K}$ to prevent recondensation of the reactants in the vapor phase. The width of the CNT fibers ranged from 10 to $25 \mu \mathrm{m}$, and the diameter of the individual CNTs ranged from 25 to $35 \mathrm{~nm}$.

\section{Electroplating}

Electroplating was carried out in an electrolytic bath that consisted of copper sulfate $(200 \mathrm{~g} / \mathrm{L})$, and $40 \mathrm{~mL}$ of $\mathrm{H}_{2} \mathrm{SO}_{4}$ was added to $1 \mathrm{~L}$ of aqueous copper sulfate. The bath temperature was maintained at room temperature. Electrodeposition was performed in a two-electrode system that was equipped with a direct current power supply. The electrolytic CNT fibers acted as the cathode, and a titanium basket with copper spheres was the anode. Electrodeposition was conducted at an applied current density of $\sim 0.03 \mathrm{~A} / \mathrm{cm}^{2}$. The amount of copper deposited increased with increasing current injection time. The sulfuric acid dip process was not performed in our experiment to avoid introducing sulfide contaminants.

\section{Structural properties}

The CNT-Cu hybrid fibers were characterized using an SEM and EDX system (Nova NanoSEM 450, FEI). The cross-sections of the fibers were characterized with a 200$\mathrm{kV}$ field emission TEM system (JEM-2100F HR, JEOL, Ltd.) after sampling by an FIB system (Helios NanoLAB 650, FEI).

\section{Electrical and magnetotransport properties}

The transport properties of the $\mathrm{Cu}$-electroplated CNTs were studied in standard four-terminal configurations using the delta mode of a Keithley 6221 current source and $2182 \mathrm{~A}$ nanovoltmeter. All measurements were performed in a $14 \mathrm{~T}$ superconducting magnet (Oxford Instruments) at temperatures ranging from 1.6 to $300 \mathrm{~K}$. The resistance was measured in the ohmic regime, and the electrical power was kept below $1 \mu \mathrm{W}$ to avoid potential electrical heating. The samples were attached to the printed circuit board substrate at the end of a sample stick. Hence, the magnetic field was applied perpendicular to the direction of the fiber axis.

\section{Results}

Preparation and characterization of the CNT-Cu microfibers

Continuous CNT fibers were prepared via direct spinning using a floating catalyst CVD method ${ }^{14}$. Figure 1a shows a cross-sectional scanning electron microscopy (SEM) image of a representative spun fiber, which was cut using a focused ion beam (FIB). The CNT fibers usually form flattened or rolled ribbon-like structures rather than cylindrical shapes. The CNTs inside the fiber are sparsely populated, and there are many nanometer-scale voids. Figure $1 \mathrm{~b}$ illustrates the $\mathrm{Cu}$ electrochemical deposition process and shows a photograph of long CNT fibers that are mounted on a holder. The fibers were used as the cathode for electroplating. The detailed conditions of the direct spinning and electroplating processes are described in the Methods section.

Figure 1c displays schematic drawings and SEM images of a spun $\mathrm{CNT}$ fiber and $\mathrm{CNT}-\mathrm{Cu}$ microfibers prepared at various deposition times from 10 to $60 \mathrm{~s}$. Note that the amount of deposited $\mathrm{Cu}$ cannot be precisely controlled by the deposition time and varies significantly under the diverse conditions of the acidic copper sulfate solution with different CNT fibers. Moreover, even in a single fiber, the deposited $\mathrm{Cu}$ thickness is not uniform and is 


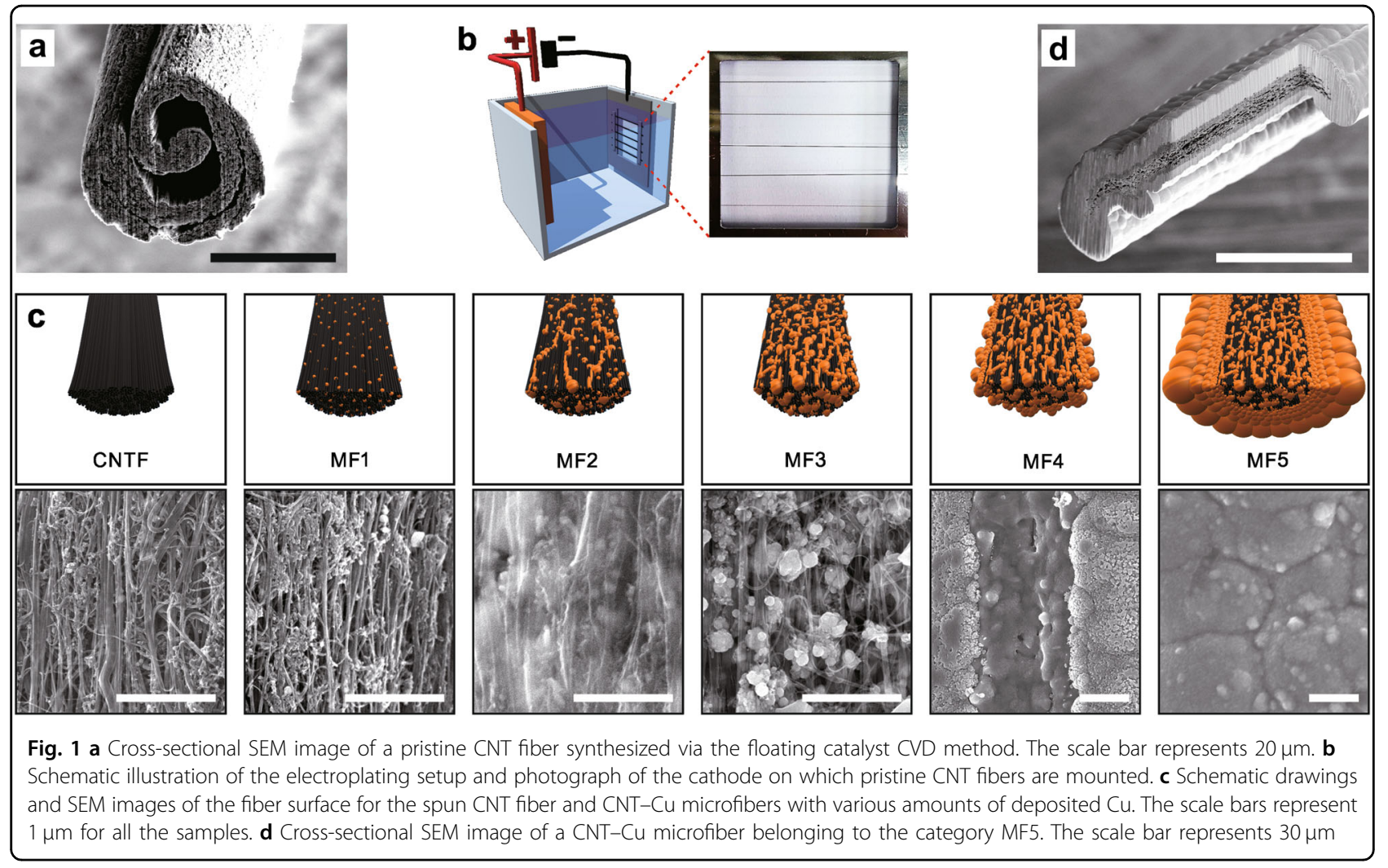

often thicker at the edge near the clamps rather than in the center because of the potential difference along the fiber. Therefore, we categorized the samples not by deposition time but by the amount of deposited $\mathrm{Cu}$, which was estimated via the SEM images. For the electrical measurements, the $\mathrm{CNT}-\mathrm{Cu}$ fiber samples were cut into small pieces approximately $2-3 \mathrm{~mm}$ in length. After the measurements, all the $\mathrm{CNT}-\mathrm{Cu}$ microfiber samples were categorized into five groups of MF1-MF5 based on the SEM inspection.

In the first stage of deposition, nanometer-scale $\mathrm{Cu}$ particles appear on the surface of the CNTs (MF1). Nucleation occurs both on the surface and inside the $\mathrm{CNT}$ fiber. Later, $\mathrm{Cu}$ grains grow from these seeds. The CNTs are covered with $\mathrm{Cu}$, and the voids become filled. Now, the CNT-Cu microfiber shows a smooth surface morphology without exhibiting small seeds (MF2). Note that this $\mathrm{Cu}$ structure formed inside the CNT fiber is not easily oxidized in ambient conditions, which was confirmed by X-ray photoelectron spectroscopy (data not shown). Further electrochemical deposition causes additional seeding on the fiber surface, and larger particles appear (MF3). The particles grow and merge with each other; finally, the $\mathrm{Cu}$ grains fully cover the fiber and form a core-shell cylinder (MF4 and MF5). The $\mathrm{Cu}$ grain size on the fiber surface increases with increasing deposition time, i.e., $\sim 1 \mu \mathrm{m}$ for MF4 and $\sim 2 \mu \mathrm{m}$ for MF5, as depicted in Fig. 1c. A cross-sectional SEM image of an MF5 fiber is shown in Fig. 1d. The image was obtained from a sample in the MF5 group that was etched using an FIB. Even after the extended deposition time, the core still shows a porous structure. The outer $\mathrm{Cu}$ layer also exhibits many voids owing to the presence of polycrystalline domains.

\section{Ampacity measurements}

The ampacity, which is the maximum current density a material can carry before breakdown, was measured for the CNT and CNT-Cu fibers in a vacuum chamber. The samples were mounted and electrically contacted using two metal "duck" clamps with flat beaks. The measurement setup can be found in Figure S1 in the Supporting Information. Figure $2 \mathrm{a}$ shows the current-voltage $(I-V)$ characteristics on a logarithmic scale of current that were obtained from the ampacity measurements. A linear scale plot is shown in the inset of Fig. 1a. The ampacity is obtained using the relation $J_{\max }=I_{\max } / A_{\mathrm{cs}}$, where $I_{\max }$ is the maximum current before breakdown and $A_{\mathrm{cs}}$ is the cross-sectional area of the fiber. After the measurements, the broken fibers were cut by a FIB, and cross-sectional SEM images were captured. The $A_{\mathrm{cs}}$ value was estimated using the sample dimensions determined from the SEM image of each sample. The calculated ampacity is plotted as solid circles in Fig. 2b. Note that the calculated ampacity values are underestimated because the 

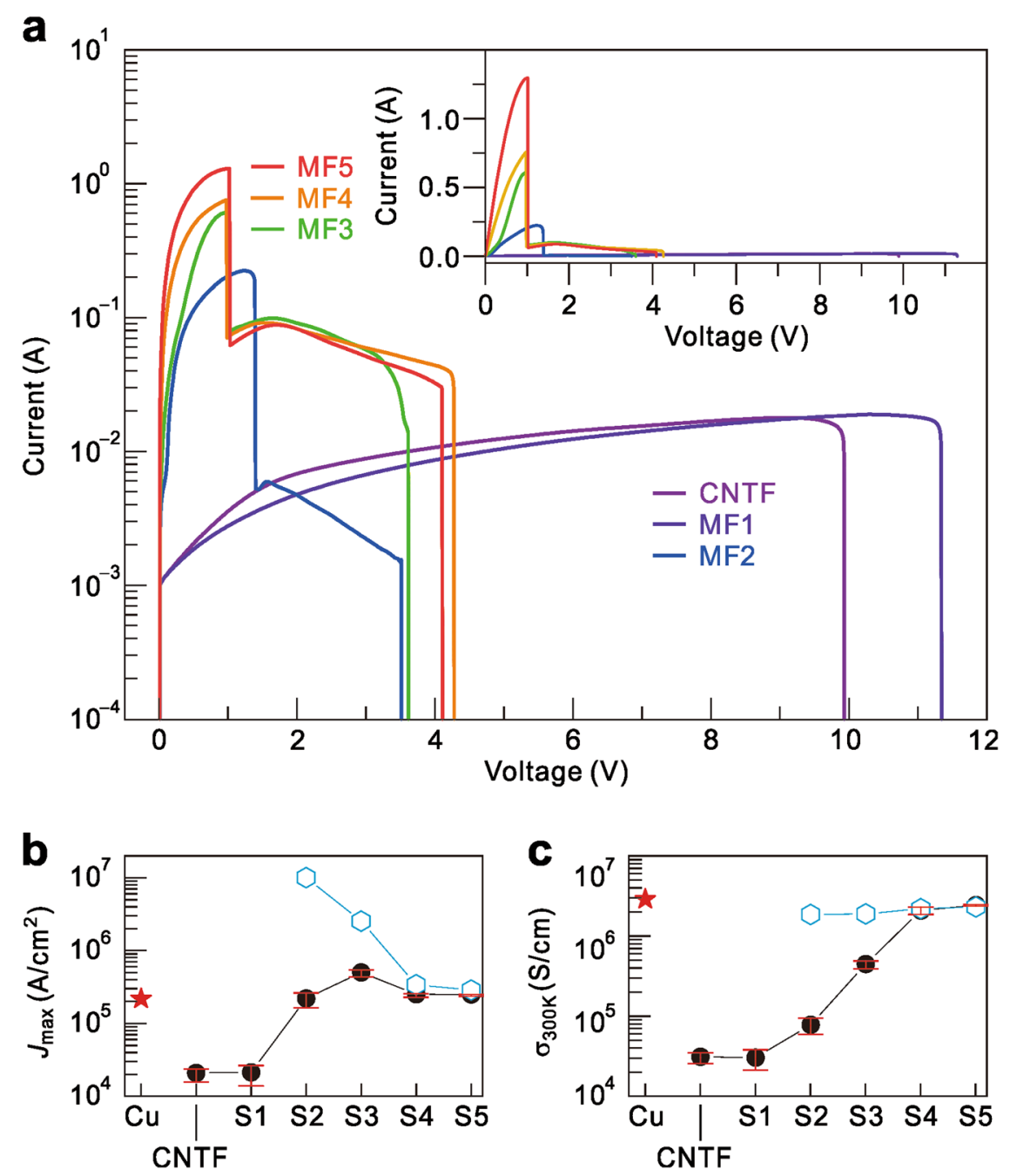

Fig. 2 Ampacity of the CNT-Cu fibers. a Logarithmic scale of the I-V characteristics obtained from the ampacity measurements for the pristine CNT fiber and CNT-Cu microfibers. The inset shows the linear scale data. b Ampacities of the CNT-Cu fibers (solid circles) and effective ampacities through the Cu conduction paths (open hexagons) of various samples. The ampacity of each sample was estimated by a calculation using the maximum current data recorded in Fig. $4 a$ and the cross-sectional areas obtained from the cross-sectional SEM images. The estimation of the effective ampacity is described in the text. The red star denotes the ampacity measured for a pure Cu wire. $\mathbf{c}$ Electrical conductivities of different samples at $300 \mathrm{~K}$ (solid circles) and effective conductivities of the Cu conduction paths (open hexagons). The electrical conductivity measured for a pure $\mathrm{Cu}$ wire is denoted by a red star

estimation of $A_{\text {cs }}$ does not consider the nanometer-scale voids present between the individual CNTs.

In the $I-V$ characteristics of the pristine CNT fiber with increasing bias voltage, the current increases linearly at low voltages and saturates at higher voltages. This saturation behavior is attributed to electron-phonon coupling ${ }^{15,16}$. At approximately $10 \mathrm{~V}$, the current drops to zero, and the maximum current, $I_{\max }$, at breakdown is $\sim 18 \mathrm{~mA}$. The ampacity of the pristine CNT fiber is estimated to be $\sim 1.78 \times 10^{4} \mathrm{~A} / \mathrm{cm}^{2}$. This value is much lower than the ampacity of the individual metallic CNTs, i.e., $\sim 10^{9} \mathrm{~A} / \mathrm{cm}^{217}$, owing to the many contact barriers present in the conduction path of the long CNT fiber. The conductivity of the pristine CNT fiber, which was measured via a four-probe method using Au microwires and silver paint, is also low $\left(\sim 3 \times 10^{3} \mathrm{~S} / \mathrm{cm}\right)$. The conductivities of the samples at room temperature are shown as solid circles in Fig. 2c. Note that neither the substrate nor the dielectric encapsulation layer is used for the measurement, which enhances heat dissipation and suppresses interfacial diffusion ${ }^{18,19}$.

The $J_{\max }$ value of MF1 is similar to that of the pristine $\mathrm{CNT}$ fiber even though decoration by $\mathrm{Cu}$ particles is expected to reduce the contact resistance between CNTs and enhance the efficiency of heat dissipation at the contacts. This result is likely due to degradation of the 
CNTs during the electrochemical process in an acidic solution. The decreased conductivity of MF1 compared with that of the pristine CNT fibers also supports this assumption. With further increases in the electroplating time, $J_{\max }$ is significantly enhanced up to $\sim 4.89 \times 10^{5} \mathrm{~A} /$ $\mathrm{cm}^{2}$ (MF3). This value is approximately 30 times higher than that of the pristine CNT fibers and 2.25 times higher than that of a pure $\mathrm{Cu}$ wire $\left(2.17 \times 10^{5} \mathrm{~A} / \mathrm{cm}^{2}\right)$, which was measured on a pure $\mathrm{Cu}$ microwire with a diameter of $15 \mu \mathrm{m}$. The enhancement in the current-carrying capacity may be explained by the reduction in Cu electromigration in the $\mathrm{CNT}-\mathrm{Cu}$ fiber owing to the increase in activation energy for $\mathrm{Cu}$ diffusion on the CNT surface. When the $\mathrm{CNT}$ fiber is covered by an outer $\mathrm{Cu}$ layer (MF4 and MF5), the ampacity starts to decrease with increasing deposition time.

It is also notable that the $I-V$ characteristics of the $\mathrm{CNT}-\mathrm{Cu}$ fibers behave differently from that of the pristine CNT fiber in Fig. 2a. For the pristine CNT fiber and MF1, the current abruptly drops to zero at high voltages. In contrast, for the other fibers, i.e., MF2-MF5, two-step failure was observed. The curve for MF3 is shown in Fig. $2 \mathrm{a}$ as an example. At approximately $1 \mathrm{~V}$, the current drops from $\sim 700$ to $\sim 80 \mathrm{~mA}$. After the first step of the drop, the current slightly increases with increasing voltage and then begins to decrease again at $\sim 2 \mathrm{~V}$. Then, the current slowly decreases in several steps before the final failure occurs at $\sim 3.6 \mathrm{~V}$.

\section{Temperature-dependent electrical resistivity}

To understand the ampacity behavior and heat dissipation mechanism of the $\mathrm{CNT}-\mathrm{Cu}$ microfibers, we investigated the temperature and magnetic field dependence of electrical transport. Figure $3 a-d$ displays the temperature-dependent resistivity $(\rho(T))$ of the samples discussed in this paper in the temperature $(T)$ range from 1.6 to $300 \mathrm{~K}$. In Fig. 3a, the resistivity is normalized by the room-temperature $(300 \mathrm{~K})$ resistivity to compare the resistivity behaviors of different samples. Each curve shows various signatures. The resistivity curves for the pristine CNT fiber and MF1 show similar temperaturedependent behaviors. The samples exhibit a metallic trend $(\partial \rho / \partial T>0)$ at high temperatures and a non-metallic trend $(\partial \rho / \partial T<0)$ at low temperatures, while the transition occurs at $\sim 115 \mathrm{~K}(\sim 140 \mathrm{~K})$ for the CNT fiber (MF1). This crossover behavior has been observed in CNT fibers ${ }^{13}$, iodine-doped $\mathrm{CNTs}^{20,21}$, and doped polymers ${ }^{22}$ and can be explained by a heterogeneous model that involves extended metallic regions that are separated by small barriers $^{23}$. The heterogeneous model is expressed by the following equation:

$$
\rho(T)=A_{\exp }\left(-\frac{T_{m}}{T}\right)+B_{\exp }\left(\frac{T_{b}}{T_{s}+T}\right)
$$

where the first term corresponds to quasi-onedimensional metallic conduction and the second term represents fluctuation-induced tunneling conduction between metallic regions that are separated by electrical barriers. $T_{\mathrm{m}}$ provides the phonon energy $\left(k_{\mathrm{B}} T_{\mathrm{m}}\right)$ with the wave vector $2 k_{\mathrm{F}}$ that can backscatter carriers. $T_{\mathrm{b}}$ is the temperature at which the amplitude of the fluctuations is equivalent to the barrier height. $T_{\mathrm{s}}$ is the temperature at which thermally activated conduction over the potential barrier starts to occur. Therefore, the ratio of the characteristic temperatures, $T_{\mathrm{b}} / T_{\mathrm{s}}$, implies the importance of thermal activation for quantum tunneling in the conduction mechanism and reflects the effective barrier height in the absence of fluctuations at zero temperature. The model accurately fits the curves for the CNT fiber and MF1, as shown in Fig. 3b. The fitting parameters are summarized in Table 1 . The ratio of $T_{\mathrm{b}} / T_{\mathrm{s}}$ for MF1 is slightly higher than that for the pristine CNT fiber by approximately $10 \%$. This result also suggests that nanometer-sized $\mathrm{Cu}$ particles do not effectively reduce the barrier height, and the electrolyte may even increase the height during the electroplating process.

The resistivity of the core-shell cylinders (MF4 and MF5) shows a different temperature dependence. It monotonically decreases during the cool-down of the samples across the whole temperature measurement range, as shown in Fig. 3d. This typical metallic behavior indicates that the majority of the current flows through the outer $\mathrm{Cu}$ layer. At low temperatures, the resistivity saturates at a non-zero value, which is termed the residual resistivity $\left(\rho_{0}\right)$. The $\rho_{0}$ value measured for a commercial $\mathrm{Cu}$ microwire is $\sim 6.11 \times 10^{-8} \Omega \cdot \mathrm{cm}$, whereas those of MF4 and MF5 are two orders of magnitude higher. This result implies that charge transport in the core-shell cylinders is disturbed by strong structural scattering. The RRRs, $\left(\rho_{300 K} / \rho_{0} \approx \rho_{300 K} / \rho_{1.6 K}\right)$ of MF4, MF5 and the $\mathrm{Cu}$ wire were found to be approximately 2.6, 3.1 and 56.7, respectively. The $\rho(T)$ value of non-magnetic metals dominantly arises from electron-phonon interactions as a function of temperature ${ }^{25}$, which is given by the BlochGruneisen (BG) formula,

$$
\rho(T)=\rho_{0}+\alpha\left(\frac{T}{\Theta_{R}}\right)^{n} \int_{0}^{\frac{\Theta_{R}}{T}} \frac{x^{n}}{\left(e^{x}-1\right)\left(1-e^{-x}\right)} d x,
$$

where $\Theta_{\mathrm{R}}$ and $\alpha$ are the Debye temperature and electronphonon interaction constants, respectively. The exponent $n$ is normally fixed at 2,3 , or 5 depending on the material; e.g., for $\mathrm{Cu}$, it is fixed at $n=5$. The light blue narrow lines in Fig. $3 \mathrm{~d}$ are the fittings of the experimental data via the BG formula. The fitting parameters are $\rho_{0}=2.06 \Omega \cdot \mathrm{cm}, \alpha$ $=12.5 \mu \Omega \cdot \mathrm{cm}$ and $\Theta_{\mathrm{R}}=292 \mathrm{~K}$ for MF4 and $\rho_{0}=1.48$ $\mu \Omega \cdot \mathrm{cm}, \alpha=13.1 \mu \Omega \cdot \mathrm{cm}$ and $\Theta_{\mathrm{R}}=295 \mathrm{~K}$ for MF5. The 

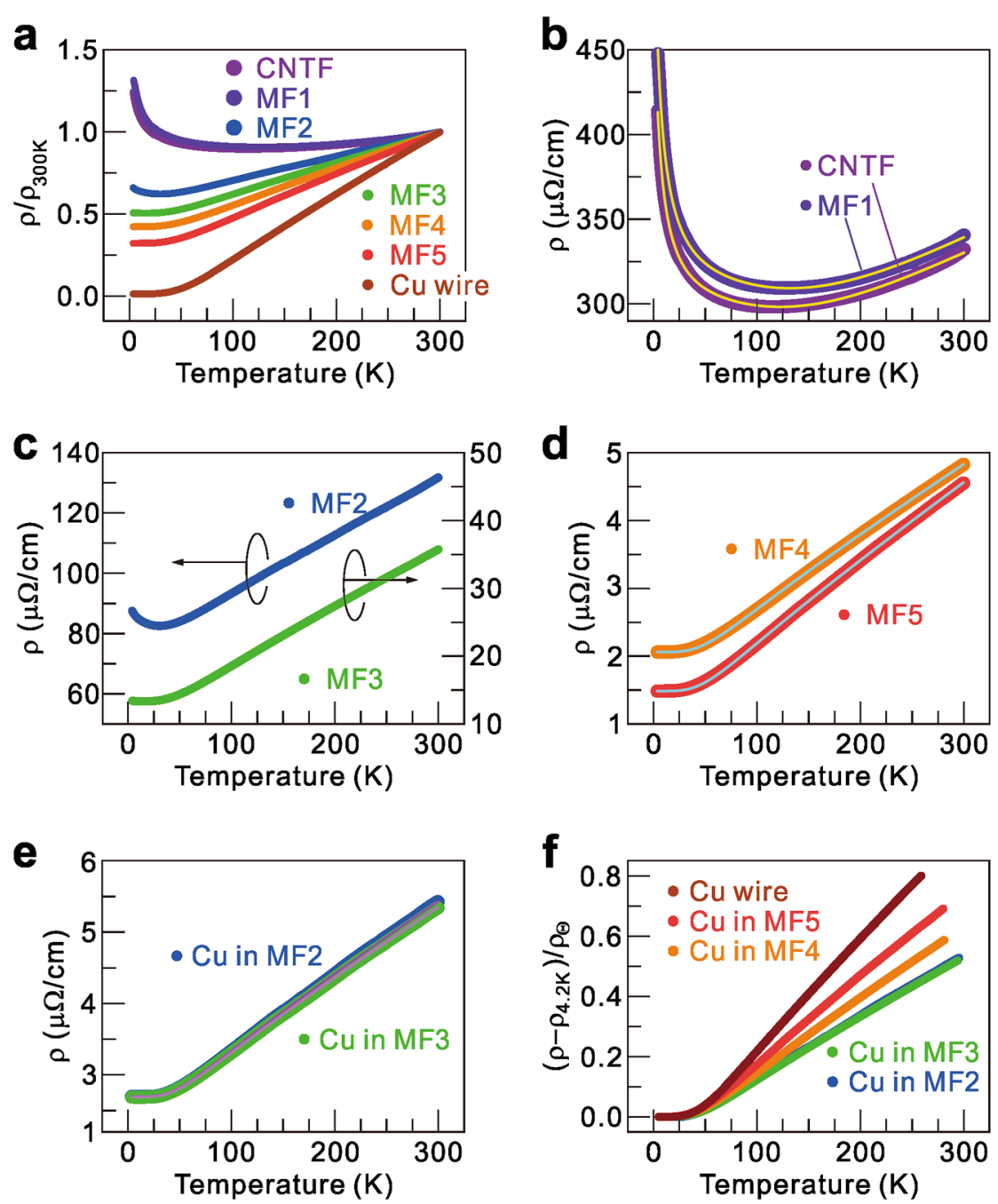

Fig. 3 Analysis of the temperature-dependent electrical transport. a Normalized resistivity $\left(\rho / \rho_{300 \mathrm{~K}}\right)$ observed in the temperature range of $1.6 \mathrm{~K}$ $<T<300 \mathrm{~K}$. b Temperature-dependent resistivity of the CNT fiber and MF1. The yellow solid lines are the fits to the heterogeneous model. $\mathbf{c}$ Temperature-dependent resistivities of MF2 and MF3. $\mathbf{d}$ Temperature-dependent resistivities of MF4 and MF5. The light blue solid lines are the fits by the BG function. e Effective contribution of the Cu fibrils embedded in MF2 and MF3 to the temperature-dependent resistivity. f Scaling plot of $\left(\rho-\rho_{0}\right) / \rho_{\ominus}$ as a function of $T / T_{\Theta}$

close match and fit of the data indicate that electrical transport is dominated by electron-phonon interactions. Note that the fitting parameters for MF4 are close to the previously reported values for nanocrystalline $\mathrm{Cu}^{24}$. MF5 exhibits a higher RRR and lower $\rho_{0}$ value, which means that grain boundary scattering is suppressed because of the presence of larger grains. This result again confirms that the $\mathrm{Cu}$ grains are nanometer-sized near the CNTs and that the grains become larger as $\mathrm{Cu}$ is further deposited.

The $\rho(T)$ curves of MF2 and MF3 show metallic behavior similar to the curves of MF4, MF5 and $\mathrm{Cu}$ at high temperatures except for a small turn-up at low temperatures. There are two possible scenarios that explain the strong metallic behavior and lower resistivity values of
MF2 and MF3 compared with those of the CNT fiber and MF1. First, we consider a situation in which more $\mathrm{Cu}$ particles are present between individual CNTs than in MF1. In this case, the effective barrier height would be reduced, and the $\rho(T)$ curves of MF2 and MF3 would also be fitted by the heterogeneous model with smaller $T_{\mathrm{b}} / T_{\mathrm{s}}$ values. However, none of the curves are reasonably fit by the model. Excluding the first scenario, we conclude that $\mathrm{Cu}$ effectively grows through the voids inside the CNT fiber structure and forms non-broken conduction paths. Given that the CNT fiber and the filled $\mathrm{Cu}$ fibrils act as two parallel resistors, the increase in resistivity at low temperature for MF2 and MF3 can be understood as a contribution to the shooting resistance of the CNT fibers, as shown in Fig. 3b. For a more quantitative analysis, we 
Table 1 Parameters for fitting the CNT microfiber and MF1 data using a heterogeneous model

\begin{tabular}{lllll}
\hline & $T_{\mathbf{m}}$ & $T_{\mathbf{b}}$ & $T_{\mathbf{s}}$ & $T_{\mathbf{b}} / T_{\mathbf{s}}$ \\
\hline CNTF & 649.13 & 4.22 & 8.04 & 0.52 \\
MF1 & 673.13 & 5.47 & 9.48 & 0.58 \\
\hline
\end{tabular}

calculated the conductance of MF2 and MF3 by combining the conductance values of the pristine CNT fibers and MF5 using the formula $A_{\mathrm{CNT}-\mathrm{Cu}} \cdot \sigma_{\mathrm{CNT}-\mathrm{Cu}}=$ $A_{\mathrm{CNTF}} \cdot \sigma_{\mathrm{CNTF}}+A_{\mathrm{Cu}} \cdot \sigma_{\mathrm{Cu}}$, where ${ }_{A x}$ and $\sigma_{x}$ are the crosssectional area and conductivity, respectively, of sample $x$. For the calculation of the $A_{\mathrm{CNTF}} \cdot \sigma_{\mathrm{CNTF}}$ term, the CNT fiber data that are shown in Fig. 3b were used. The term $\sigma_{\mathrm{Cu}}$ was obtained from the MF4 data after the contribution of the CNTs $\left(A_{\mathrm{CNTF}} \cdot \sigma_{\mathrm{CNTF}}\right)$ was subtracted. The data of MF2 and MF3 $\left(A_{\mathrm{CNT}-\mathrm{Cu}} \cdot \sigma_{\mathrm{CNT}-\mathrm{Cu}}\right)$ were fitted using only $A_{\mathrm{Cu}}$, which represents the effective cross-sectional area of the electroplated $\mathrm{Cu}$ fibrils in the $\mathrm{CNT}-\mathrm{Cu}$ hybrid structure. First, the $\mathrm{Cu}$ microwire data from Fig. 3a were used for the value of $\sigma_{\mathrm{Cu}}$, but the fitting curve significantly deviated from the data. This result implies that the electrical properties of the $\mathrm{Cu}$ fibrils formed inside the CNT fiber are distinct from those of conventional $\mathrm{Cu}$, which is most likely due to the difference in grain size. The MF5 data exhibit a better fit than the $\mathrm{Cu}$ microwire data, and the MF4 data provide the best fit among the MF4, MF5 and $\mathrm{Cu}$ wire samples. The data are provided in Figure S2 in the Supporting Information. From the fit of MF4, the effective areas of MF2 and MF3 were estimated to be 2.3 and $23 \mu \mathrm{m}^{2}$, respectively. Using the fitting parameters, we can separate the contributions of $A_{\mathrm{Cu}} \cdot \sigma_{\mathrm{Cu}}$ and $A_{\mathrm{CNTF}} \cdot \sigma_{\mathrm{CNTF}}$. From this analysis, we can extract the effective resistivity of the $\mathrm{Cu}$ fibrils in MF2 and MF3 as $\rho_{\mathrm{Cu}}(T)=1 / \sigma_{\mathrm{Cu}}(T)$. The resistivity data are plotted in Fig. 3e. Both sets of data are fit well using the BG formula (narrow purple lines). From the fitting of the effective resistivity of the $\mathrm{Cu}$ fibrils, the obtained RRR value is 2.0 for both MF2 and MF3. The residual resistivity values for MF2 and MF3 are 2.69 and $2.59 \mu \Omega \cdot \mathrm{cm}$, respectively. A scaling equation was obtained by dividing Eq. 2 by the resistivity at the Debye temperature $\rho\left(\Theta_{\mathrm{R}}\right)^{25}$. The dimensionless parameter $\alpha / \rho\left(\Theta_{\mathrm{R}}\right)$ is kept constant at 4.225 for bulk metals, and the plots of $\left(\rho(T)-\rho_{0}\right) / \rho\left(\Theta_{R}\right)$ as a function of $T / \Theta_{\mathrm{R}}$ fall into a single curve. When the material is nanocrystalline, $\left(\rho(T)-\rho_{0}\right) / \rho\left(\Theta_{\mathrm{R}}\right)$ deviates from the bulk data, and $\alpha / \rho\left(\Theta_{\mathrm{R}}\right)$ is below the value of the bulk $^{24}$. The scaling plots for the data of the metallic samples, i.e., MF4, MF5 and bulk $\mathrm{Cu}$ wire, and the extracted data of the Cu fibrils formed in MF2 and MF3, are displayed in Fig. 3f. The scaling parameter $\alpha / \rho\left(\Theta_{\mathrm{R}}\right)$ is 4.213 for the $\mathrm{Cu}$ microwire, which is consistent with the value of bulk materials. For the other samples, the $\alpha / \rho\left(\Theta_{\mathrm{R}}\right)$ value sequentially decreases from 3.766 for MF5 to 3.342 for MF2. This result suggests that $\mathrm{Cu}$ with nanometerscale grains is deposited near the CNT fiber surface and that the grain size increases as the thickness of the deposited $\mathrm{Cu}$ layer increases, as shown in Fig. 1c. The smallest scaling parameter for the $\mathrm{Cu}$ fibrils embedded in MF2 and MF3 implies the smallest grain size.

\section{MR at low temperatures}

The MR that is measured at low temperatures is also a manifestation of the nanocrystalline nature of the $\mathrm{Cu}$ fibrils that are formed inside the CNT fiber. The MR is defined as $[R(H)-R(0)] / R(0)$, where $H$ is the magnetic field and $R(0)$ is the zero magnetic field resistance. The CNT fiber samples MF1, MF2 and MF3 show a negative MR that exhibits two-dimensional weak localization at low temperatures $<100 \mathrm{~K}$, which is consistent with a previous report on spun CNT fibers ${ }^{26}$. These data are shown in Figure S3 and Figure S4 in the Supporting Information. Unlike the temperature-dependent resistivity, the MR at low $T$ is dominated by conduction through the CNT networks not only for the bare CNT fiber and MF1 but also for MF2 and MF3. This conduction is because the MR of the $\mathrm{Cu}$ fibrils that formed in MF2 and MF3 is small because of their nanocrystalline nature. Figure 4 displays the MR results of MF4 (a), MF5 (b) and the $\mathrm{Cu}$ microfiber (c). Classically, a non-magnetic metal with an open Fermi surface exhibits a quadratic dependence of the MR on $H,[R(H)-R(0)] / R(0) \propto(\mu H)^{2}$, where $\mu$ is the charge carrier mobility. We observed that the MR of MF5 is smaller than that of the $\mathrm{Cu}$ wire or bulk copper ${ }^{27}$. As the residual resistivity increases or the RRR decreases, the MR decreases. A reduction in MR is observed in gold film and bulk copper, which results from the enhancement of the scattering sources, such as dislocations, grain boundaries, and impurities ${ }^{28,29}$. The RRR values of MF4, MF5 and the $\mathrm{Cu}$ microwire are 2.6, 3.1 and 56.7, respectively. Additionally, the RRR value of the $\mathrm{Cu}$ fibrils that formed in MF2 and MF3 is 2.0. This result indicates that the electroplated $\mathrm{Cu}$ contains more scattering sources than bulk $\mathrm{Cu}$, which is consistent with our conclusion that the electroplated $\mathrm{Cu}$ near the individual CNTs exhibits nanosized grains. The MR values for MF4, MF5 and the $\mathrm{Cu}$ microwire are $0.001,0.003$ and 0.51 , respectively. For the samples with smaller domains, MF2 and MF3, the MR contribution of the $\mathrm{Cu}$ fibrillar conduction paths is small; therefore, these samples also exhibit weak localization behavior similar to that of the pristine CNT fiber at temperatures lower than $100 \mathrm{~K}$ (Figure S3). Note that regarding the temperature-dependent resistivity, these samples show metallic behavior, unlike the CNT fiber and MF1. 

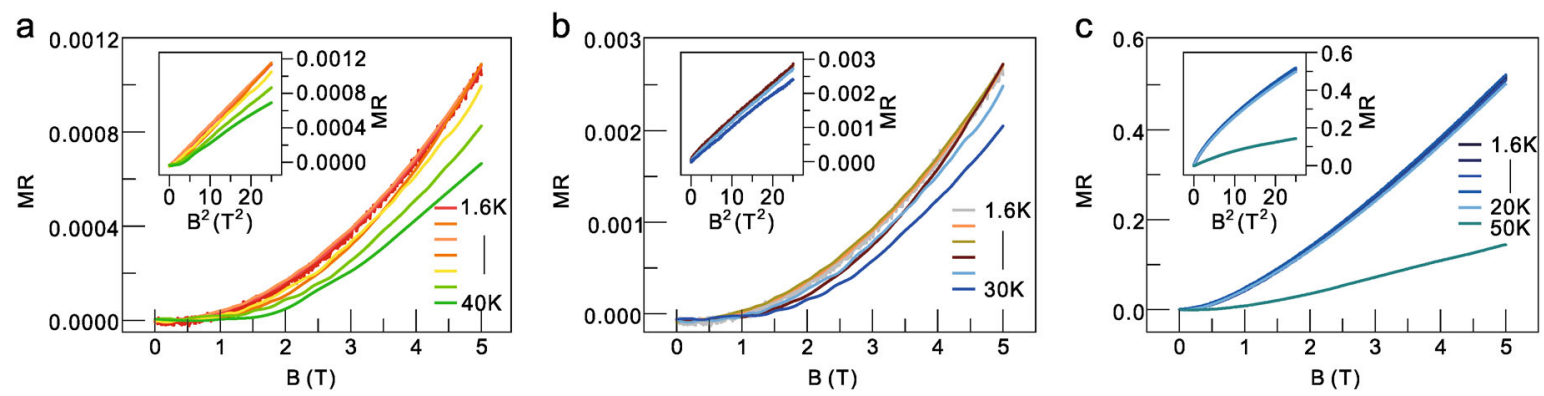

Fig. 4 Magnetoresistance as a function of the magnetic field (B) at various temperatures for MF4 (a), MF5 (b) and the pure Cu wire (c). The insets are the plots of MR as a function of $B^{2}$

\section{Discussion}

Failure mechanism of the CNT-Cu hybrid fibers

We first discuss the two-step failure of the $\mathrm{CNT}-\mathrm{Cu}$ microfibers shown in Fig. 2a. Typically, the failure of the $\mathrm{Cu}$ line originates from electromigration because of the displacement of $\mathrm{Cu}$ atoms in response to an electric field ${ }^{30}$.

In CNT fibers, current-induced Joule heating damages and ultimately breaks the fibers ${ }^{31,32}$. Figure 5a shows an SEM image of MF5 after electrical breakdown following the ampacity measurement. At the broken tip of the fiber, molten $\mathrm{Cu}$ is retracted, and the original CNT fiber structure is exposed. For the core-shell cylinder samples, the majority of the current flows through the outer $\mathrm{Cu}$ layer, as determined via the transport measurements. Under a high current bias, the outer $\mathrm{Cu}$ layer becomes disconnected through electromigration beginning at the grain boundaries, which is similar to the failure in bulk $\mathrm{Cu}$. Note that the ampacity values for MF4 and MF5 are comparable with that of the bulk $\mathrm{Cu}$ microwire $\left(\sim 2 \times 10^{5}\right.$ $\mathrm{A} / \mathrm{cm}^{2}$ ), as shown in Fig. 2b. These results imply that the $\mathrm{Cu}$ fibril structures inside the CNT fiber core maintain their shape after the first failure and contribute to the conduction. Prior to the second failure, the current gradually decreases as the voltage increases. Such behavior is distinct from that of the outer $\mathrm{Cu}$ shell, in which the current abruptly drops. After the final failure, the $\mathrm{Cu}$ fibrils and the CNT fiber membrane are disconnected. However, molten $\mathrm{Cu}$ behaves differently from the $\mathrm{Cu}$ that is outside the CNT fiber, where it retracts from the hot spot. Instead, the molten $\mathrm{Cu}$ merges and forms microsized particles, which are randomly distributed between the individual CNTs, as shown in Fig. 5b. For comparison, the cross-sectional image of the as-prepared MF5 sample before the ampacity measurement is shown in Fig. 5c.

Thus, we believe that the $\mathrm{Cu}$ fibrils that are entangled with the CNTs inside the microfiber core form an efficient structure with a high current-carrying capacity. We suggest three synergetic effects of the structure, as follows. (1) The CNTs can efficiently dissipate heat from the $\mathrm{Cu}$ fibrils because of their high thermal conductivity $(\kappa \sim 3000 \mathrm{~W} / \mathrm{m}$ $\mathrm{K})^{33}$. The $\mathrm{Cu}$ fibrils can conduct current if the local temperature is below the melting temperature of $\mathrm{Cu}$. For bulk $\mathrm{Cu}$, the temperature is approximately $1358 \mathrm{~K}$; for nanocrystalline $\mathrm{Cu}$, it is lower because of the melting point depression that is associated with the increased surface to volume ratio; thus, $T_{\mathrm{m}}<1273 \mathrm{~K}$ for nanocrystalline $\mathrm{Cu}^{34}$. (2) Mechanical interlocking between $\mathrm{Cu}$ and the CNTs can suppress the surface diffusion of $\mathrm{Cu}$. The observation of microscale molten particles that are randomly distributed inside the fiber core after failure implies the existence of interlocking friction (Fig. 5b). We investigated the interfacial bonding strength between $\mathrm{Cu}$ and the CNTs through tensile strength measurements of the fibers (data shown in Figure S5 in the Supporting Information). Depending on the samples, the trend in the tensile strength is similar to that of the ampacity. MF3 shows the highest tensile strength among the measured samples, and MF4-MF5 show a reduction in tensile strength. (3) Moreover, the $\mathrm{Cu}$ fibrillar structure itself exhibits a grain boundary location that suppresses electromigration. Although the nanocrystalline metal structure has the disadvantage of a lower melting temperature for carrying high current, this structure can be helpful when the grain boundary structures are controlled. The CNT fiber with nanometer-size voids may serve as a template for an improved grain boundary location. A narrow metal wire with a smaller width than the grain size is often helpful for enhancing the ampacity. This is because the narrow wire forms a bamboo-like structure, which significantly reduces the number of grain triple points. The appearance of voids at the triple points are known to trigger the electromigration process. The fibrils are composed of small grains with sizes in the range from 10 to $20 \mathrm{~nm}$, as observed in the high-resolution transmission electron microscopy image and estimated from the high-resolution X-ray diffraction measurement (Figure $\mathrm{S} 6$ in Supporting information) of the as-prepared MF2 sample not subjected to the ampacity measurement (Fig. 5d). 

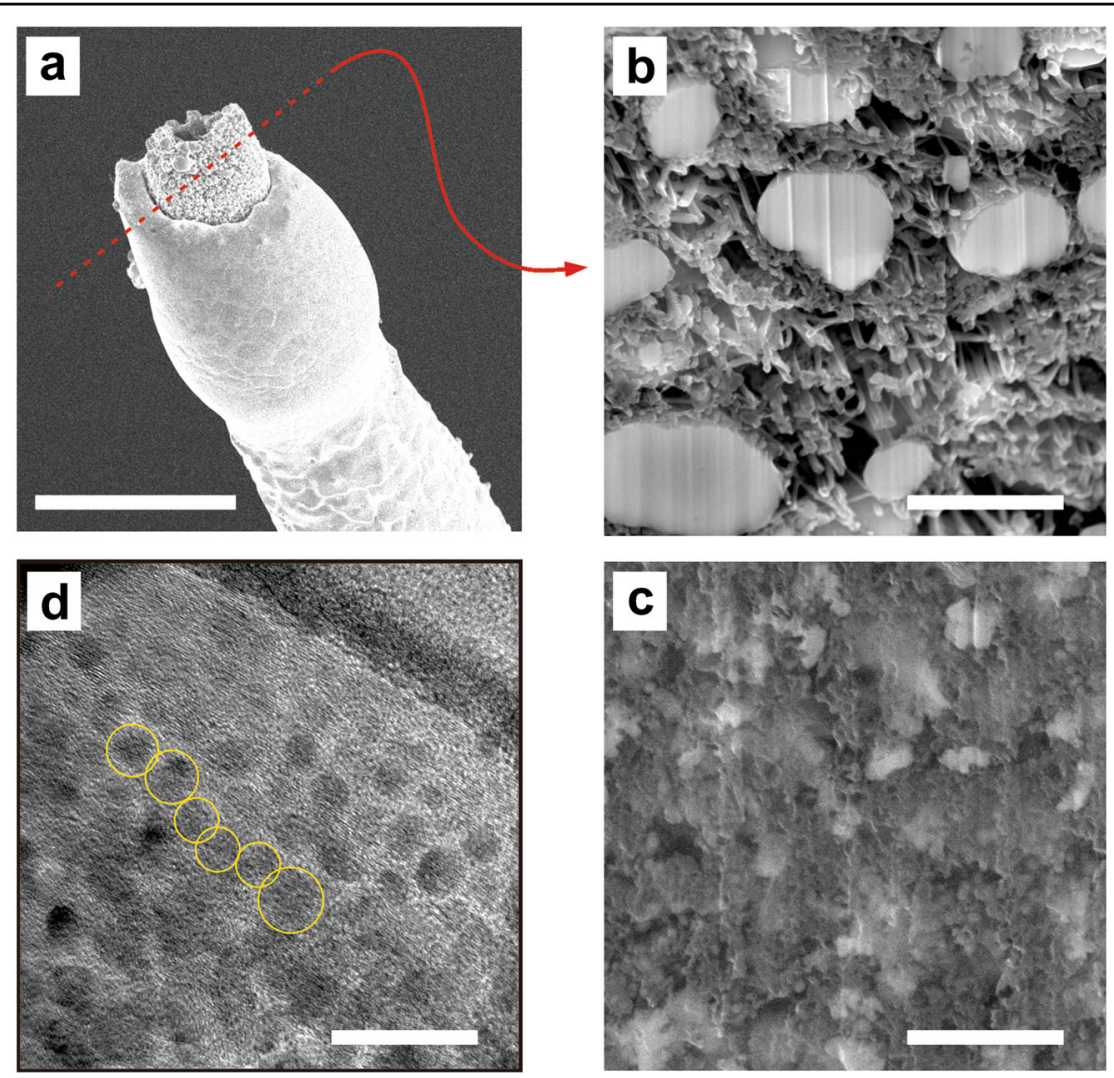

Fig. 5 a An SEM image of the MF5 sample after electrical breakdown. The scale bar represents $50 \mu \mathrm{m}$. b Cross-sectional SEM image of the MF5 sample cut along the red dashed line marked in a via a FIB. The scale bar represents $1 \mu \mathrm{m}$. c Cross-sectional SEM image of the core section of an as-prepared MF5 sample not subjected to the ampacity measurement. $\mathbf{d}$ High-magnification TEM image of the core section of an MF2 sample. The scale bar represents $20 \mathrm{~nm}$. Yellow circles indicate the Cu grains

\section{Failure mechanism of the CNT-Cu hybrid fibers}

Although the outer $\mathrm{Cu}$ layer is thin and the large particles on the fiber surface are not merged, as shown in the SEM image in Fig. 1c, the ampacity of MF3 still exhibits the highest value among the samples. Moreover, MF2 with no outer $\mathrm{Cu}$ layer shows an ampacity comparable to that of bulk $\mathrm{Cu}$. As confirmed by the transport measurement shown in Fig. 3c, the majority of the current flows through the $\mathrm{Cu}$ fibrils inside the fiber at high temperatures. However, the effective cross-sectional areas of $\sim 2.3$ and $23 \mu \mathrm{m}^{2}$ for MF2 and MF3, respectively, are small compared with that of the CNT fiber, which ranges from $\sim 180$ to $210 \mu \mathrm{m}^{2}$. Using the effective cross-sectional areas of the $\mathrm{Cu}$ fibril structures in MF2 and MF3, we calculated the effective ampacity, i.e., the maximum current that flows through the $\mathrm{Cu}$ fibrils divided by the effective area of the $\mathrm{Cu}$ fibrils. Here, we ignored the CNT fiber contribution because we assumed that the dominant conduction is through the $\mathrm{Cu}$ fibrils. The $\mathrm{Cu}$ contributions to the ampacity for MF4 and MF5 were calculated using the summation of two areas, the area of the outer $\mathrm{Cu}$ layer of the MF samples and the effective area of the $\mathrm{Cu}$ fibrils in the core region, the latter of which was estimated to be the same as that in MF3 $\left(23 \mu \mathrm{m}^{2}\right)$. The results are displayed as open diamonds in Fig. 2b. Similarly, we calculated the effective conductivity of the samples at room temperature, and the results are depicted as open diamonds in Fig. 2c. The effective ampacity of $\mathrm{Cu}$ shows a maximum value of $\sim 1 \times 10^{7} \mathrm{~A} / \mathrm{cm}^{2}$ in MF2, which is $\sim 50$ times higher than the ampacity measured for bulk $\mathrm{Cu}$. The ampacity decreases for MF3-MF5 and saturates at a value that is similar to the ampacity of bulk $\mathrm{Cu}$. Note that the effective conductivities of MF2-MF5 are similar to each other as well as to that of bulk $\mathrm{Cu}$. These values are within the range of $2-3 \times 10^{6} \mathrm{~S} / \mathrm{cm}$. The lower conductivity values of MF2 and MF3 compared with those the others are attributed to the smaller grain size of the $\mathrm{Cu}$ fibrils that are formed inside the core, as discussed above.

In summary, we report a method for producing $\mathrm{CNT}-\mathrm{Cu}$ microfibers using $\mathrm{Cu}$ electroplating on continuous CNT fibers. Temperature- and magnetic fielddependent transport measurements reveal that nanofibrillar structures are formed inside the CNT fibers. The 
$\mathrm{Cu}$ nanofibrils can conduct an approximately 50 times higher current density than a bulk $\mathrm{Cu}$ microwire owing to the controlled grain boundary location and heat dissipation by the CNTs. The material is promising for application in highly integrated microelectronics, high-power electronics, and low-mass high-power transmission cables.

\section{Acknowledgements}

This work was supported by the Korea Institute of Science and Technology (KIST) Institutional Program. D.S.L and S.M.K acknowledge support of programs through the National Research Foundation of Korea (NRF) funded by the Ministry of Science, ICT and Future Planning (NRF-2017M3A7B4049167 and NRF-2016M3A7B4905619).

\section{Authors' contributions}

S.H.L. and D.S.L. conceived of the project and designed the experiments. S.M.K. and J.H. synthesized the continuous CNT fibers. H.R. and Mina P. conducted Cu electroplating and the mechanical property measurements. J.H. and D.S.L. contributed to the ampacity measurements. Min P. and D.S.L conducted the temperature- and magnetic field-dependent measurements. A.L. performed the data fitting and analyses. S.B., T.-W.K and J.-S.H characterized the structural properties of the CNTs and copper. S.H.L. supervised the overall study. All authors contributed to the discussions about and writing of the manuscript.

\section{Conflict of interest}

The authors declare that they have no conflict of interest.

\section{Publisher's note}

Springer Nature remains neutral with regard to jurisdictional claims in published maps and institutional affiliations.

Supplementary information is available for this paper at https://doi.org/ 10.1038/s41427-018-0028-3.

Received: 22 September 2017 Revised: 19 December 2017 Accepted: 4 February 2018.

Published online: 11 April 2018

\section{References}

1. Tu, K. N., Liu, Y. \& Li, M. Effect of Joule heating and current crowding on electromigration in mobile technology. Appl. Phys. Rev. 4, 011101 (2017).

2. Bai, J. G., Zhang, Z. Z., Calata, J. N. \& Lu, G.Q. Low-temperature sintered nanoscale silver as a novel semiconductor device-metallized substrate interconnect material. IEEE Trans. Compon. Pack. Manuf. Technol. 29, 589-593 (2006).

3. Weitzel, C. E. et al. Silicon carbide high-power devices. IEEE Trans. Electron Dev. 43, 1732-1741 (1996).

4. Moore, A. L. \& Shi, L. Emerging challenges and materials for thermal management of electronics. Mater. Today 17, 163-174 (2014).

5. van Roosmalen, A. J. \& Zhang, G. Q. Reliability challenges in the nanoelectronics era. Microelectron. Reliab. 46, 1403-1414 (2006).

6. Varesi, J.\& Majumdar, A. Scanning Joule expansion microscopy at nanometer scales. Appl. Phys. Lett. 72, 37-39 (1998)

7. Roy, A. \& Tan, C. M. Very high current density package level electromigration test for copper interconnects. J. Appl. Phys. 103, 093707 (2008).

8. Baughman, R. H., Zakhidov, A. A. \& de Heer, W. A. Carbon nanotubes-the route toward applications. Science 297, 787-792 (2002).
9. Allen, M. J., Tung, V. C. \& Kaner, R. B. Honeycomb carbon: a review of graphene. Chem. Rev. 110, 132-145 (2010).

10. Subramaniam, C. et al. One hundred fold increase in current carrying capacity in a carbon nanotube-copper composite. Nat. Commun. 4, 2202 (2013).

11. Xu, G. et al. Continous electrodeposition for lightweight, highly conducting and strong carbon nanotube-copper composite fibers. Nanoscale 3, 4215-4219 (2011).

12. Fang, B. et al. Wrinkle-stabilized metal-graphene hybrid fibers with zero temperature coefficient of resistance. Nanoscale 9, 12178-12188 (2017).

13. Lekawa-Raus, A., Patmore, J., Kurzepa, L., Bulmer, J. \& Koziol, K. Electrical properties of carbon nanotube based fibers and their future use in electrical wiring. Adv. Funct. Mater. 24, 3661-3682 (2014).

14. Li, Y.-L., Kinloch, I. A. \& Windle, A. H. Direct spinning of carbon nanotube fibers from chemical vapor deposition synthesis. Science 304, 276-278 (2004).

15. Yao, Z., Kane, C. L. \& Dekker, C. High-field electrical transport in single-wall carbon nanotubes. Phys. Rev. Lett. 84, 2941-2944 (2000).

16. Park, J. Y. et al. Electron-phonon scattering in metallic single-walled carbon nanotubes. Nano Lett. 4, 517-520 (2004).

17. Wei, B. Q., Vajtai, R. \& Ajayan, P. M. Reliability and current carrying capacity of carbon nanotubes. Appl. Phys. Lett. 79, 1172-1174 (2001).

18. Liao, A. et al. Thermal dissipation and variability in electrical breakdown of carbon nanotube devices. Phys. Rev. B 82, 205406 (2010).

19. $\mathrm{Hu}, \mathrm{C} . \mathrm{K}$. et al. Reduced electromigration of $\mathrm{Cu}$ wires by surface coating. Appl. Phys. Lett. 81, 1782-1784 (2002).

20. Ahn, S. et al. Magnetotransport in iodine-doped single-walled carbon nanotubes. Phys. Rev. B 80, 165426 (2009).

21. Grigorian, L. et al. Reversible intercalation of charged iodine chains into carbon nanotube ropes. Phys. Rev. Lett. 80, 5560-5563 (1998).

22. Lee, K. et al. Metallic transport in polyaniline. Nature 441, 65-68 (2006).

23. Kaiser, A. B., Dusberg, G. \& Roth, S. Heterogeneous model for conduction in carbon nanotubes. Phys. Rev. B 57, 1418-1421 (1998).

24. Okram, G. S. \& Kaurav, N. Size-dependent resistivity and thermopower of nanocrystalline copper. J. Appl. Phys. 110, 023713 (2011).

25. Bid, A., Bora, A. \& Raychaudhuri, A. K. Temperature dependence of the resistance of metallic nanowires of diameter $\geq 15 \mathrm{~nm}$ : applicability of BlochGruneisen theorem. Phys. Rev. B 74, 035426 (2006).

26. Piraux, L., Araujo, F. A., Bui, T. N., Otto, M. J. \& Issi, J. P. Two-dimensional quantum transport in highly conductive carbon nanotube fibers. Phys. Rev. $B$ 92, 085428 (2015).

27. Berlincourt, T. G. Hall effect, magnetoresistance, and size effects in copper. Phys. Rev. 112, 381-387 (1958).

28. Oyarzun, S. et al. Transverse magnetoresistance induced by electron-surface scattering on thin gold films: experiment and theory. Appl. Surf. Sci. 289, 167-172 (2014)

29. Niewczas, M. \& Bian, Q. Influence of dislocation microstructure on low temperature magnetoresistance in copper single- and polycrystals. Mater. Des. $\mathbf{9 6}$ 203-216 (2016)

30. Lloyd, J. R. \& Clement, J. J. Electromigration in copper conductors. Thin Solid Films 262, 135-141 (1995).

31. Collins, P. G., Arnold, M. S. \& Avouris, P. Engineering carbon nanotubes and nanotube circuits using electrical breakdown. Science 292, 706-709 (2001).

32. Santini, C. A. et al. A study of Joule heating-induced breakdown of carbon nanotube interconnects. Nanotechnology 22, 395202 (2011).

33. Kim, P., Shi, L., Majumdar, A. \& McEuen, P. L. Thermal transport measurements of individual multiwalled nanotubes. Phys. Rev. Lett. 87, 215502 (2001).

34. Koper, O. \& Winecki, S. 8 specific heats and melting points of nanocrystalline materials. Nanoscale Materials in Chemistry. (Wiley, New York, NY, 2001). 